\title{
Croissance des bactéries propioniques dans le fromage : comparaison de 2 milieux de dénombrement
}

\author{
A Thierry ${ }^{1}, \mathrm{MN}$ Madec ${ }^{1}, \mathrm{R}$ Richoux ${ }^{2}$ \\ 1 Laboratoire de recherche de technologie laitière, \\ INRA, 65, rue de St-Brieuc, 35042 Rennes cedex; \\ 2 ITG, 73, rue de St-Brieuc, 35062 Rennes cedex, France
}

(Reçu le 12 janvier 1994; accepté le 11 février 1994)

\begin{abstract}
Résumé - La cinétique de croissance de flores propioniques "sauvages" ou réensemencées a été étudiée au cours de l'affinage de mini-fromages de type emmental. Le dénombrement des bactéries propioniques dans des fromages de flore de complexité variable a été effectué de manière comparative sur les milieux LGA (lithium-glycérol-agar) et YELA (yeast-extract-lactate-agar). La diversité de la population propionique a été appréciée par la comparaison des profils de restriction d'ADN en électrophorèse en champs pulsés des colonies isolées en cours d'affinage. Dans le cas de fromages fabriqués à partir de lait thermisé, la flore propionique "sauvage" était composée d'au moins 10 souches différentes (10 profils distincts sur 16). Son temps de génération en cave chaude était de $18,8 \mathrm{~h}$. Dans le cas d'une fabrication effectuée à partir de lait thermisé, microfiltré et réensemencé avec $P$ freudenreichii subsp shermanii TL 30 , seul le profil de restriction d'ADN de la souche inoculée a pu être mis en évidence à partir des bactéries propioniques isolées. Leur temps de génération en cave chaude était de $18,1 \mathrm{~h}$. Dans un fromage témoin (fabriqué à partir de lait microfiltré non réensemencé), la flore propionique "sauvage" se situait à un niveau inférieur de 3 à 6 logarithmes décimaux à la flore propionique du fromage réensemencé ; son temps de génération était de $25,6 \mathrm{~h}$. Pour ces 3 fabrications, la sélectivité du milieu LGA s'est révélée supérieure à celle du milieu YELA, ce qui s'est traduit par des dénombrements de la flore totale se développant sur LGA inférieurs de 1 à 2 logarithmes décimaux au dénombrement de la flore totale sur YELA. Malgré cette sélectivité supérieure du milieu LGA, la différenciation des colonies de bactéries propioniques et leur dénombrement sur ces 2 milieux se sont révélés de difficulté équivalente, en particulier dans les fromages au lait thermisé où une flore complexe provenant du lait se développe.
\end{abstract}

bactérie propionique / emmental / temps de génération / milieu sélectif / dénombrement

Summary - Growth of propionibacteria in cheese: comparison between 2 enumeration media. Growth of 'wild' or inoculated propionibacteria has been studied during ripening of experimental, Swiss-type cheeses. Two media have been compared regarding their ability to be used for counting propionic acid bacteria in cheeses of various complex flora: LGA (lithium-glycerol-agar) et YELA (yeastextract-lactate-agar). In order to characterize the strain variety among propionic flora, DNA restriction patterns have been established for several colonies isolated throughout ripening using pulsed 
field gel electrophoresis. Propionic flora of cheeses made with thermized milk was composed of at least 10 different strains (10 distinct patterns out of 16). Generation time of this flora during warm room ripening was equal to $18.8 \mathrm{~h}$. For cheeses made with microfiltered themized milk inoculated with pure culture of $\mathrm{P}$ freudenreichil subsp shermanii $T L 30$, only the DNA restriction pattern of the inoculated strain could be found among isolated propionibacteria. They had a generation time during warm room ripening of $18.1 \mathrm{~h}$. In cheeses made from the same milk without further inoculation with propionibacteria starter, propionic flora enumerated on LGA was markedly lower compared to propionic flora of inoculated cheeses ( 3 to 6 decimal logarithms less). For all the cheeses, a higher selectivity has been observed for LGA, on which total bacterial counts are lower than total bacterial counts on YELA (1 to 2 decimal logarithms less). However, despite this higher selectivity of LGA, the difficulty in differentiating and so enumerating propionic acid bacteria colonies was similar for both media.

\section{propionic acid bacteria / emmental / generation time / selective medium / enumeration}

\section{INTRODUCTION}

Les bactéries propioniques jouent un rôle essentiel dans l'affinage des fromages à pâte pressée cuite de type emmental, dans lesquels elles fermentent le lactate produit par les bactéries lactiques en propionate, acétate et $\mathrm{CO}_{2}$, contribuant ainsi au goût caractéristique et à l'ouverture de ces fromages. Les travaux décrivant leur cinétique de croissance au sein du fromage sont en nombre relativement réduit (Hettinga et al, 1974 ; Fryer et Peberdy, 1977 ; Gilles et al, 1983 ; Baer et al, 1993).

L'absence d'oses et les conditions d'incubation en anaérobiose confèrent un certain degré de sélectivité aux milieux généralement utilisés pour le dénombrement des bactéries propioniques, tels le YELA (yeastextract-lactate-agar, Hettinga et al, 1968) ou le TLA (trypticase-lactate-agar, Peberdy et Fryer, 1976). Cette sélectivité reste cependant insuffisante vis-à-vis des microorganismes du fromage et nécessite donc le contrôle de l'appartenance au genre Propionibacterium des colonies dénombrées (Baer et al, 1993).

Plusieurs tentatives ont été effectuées pour accroître la sélectivité du milieu YELA vis-à-vis de la flore du lait et/ou du fromage, par addition de divers composés inhibiteurs (Kurmann, 1962 ; Britz et Holzapfel, 1973 ; Peberdy et Fryer, 1976 ; Tomes et al,1991 ;
Drinan et Cogan, 1992). Si la plupart des levains lactiques sont inhibés sur ces milieux, il apparaît que les lactobacilles mésophiles ou les entérocoques s'y développent également. Madec et al ont proposé en 1993 un milieu sélectif de dénombrement des bactéries propioniques, fondé sur la résistance au lithium de ces bactéries et leur aptitude à fermenter le glycérol en anaérobiose.

Le but de ce travail était de comparer l'aptitude de ce dernier milieu slithium-glycérol-agar" (LGA) et du milieu YELA au dénombrement des bactéries propioniques "sauvages" ou réensemencées au cours de la fabrication de mini-fromages emmental de flores de complexité variable.

\section{MATÉRIELS ET MÉTHODES}

\section{Fabrication et échantillonnage}

Du lait écrémé et de la crème issus de lait de grand mélange, provenant de l'atelier Compagnie laitière européenne (Montauban de Bretagne, France) ont été mis en œuvre dans cette étude. Ils y avaient subi une thermisation $\left(63^{\circ} \mathrm{C}\right.$, 20 s). Ce lait a été partagé en 3 lots ayant subi les traitements suivants :

- lot TH: le lait thermisé réceptionné était simplement standardisé à $28 \mathrm{~g} /$ de matière grasse par ajout de crème thermisée, puis stocké $12 \mathrm{~h}$ à $4^{\circ} \mathrm{C}$ avant fabrication ; 
- lot MF (microfiltré) : le lait écrémé thermisé ètait réchauffé à $50^{\circ} \mathrm{C}$ puis épuré par microfiltration comme décrit par Trouvé et al (1991) sur une installation pilote de type Bactocatch (MFS1, Alfa Laval) équipée de membranes céramiques de diamètre moyen de pores de $1,4 \mu \mathrm{m}$; le lait microfiltré refroidi à $4^{\circ} \mathrm{C}$ était ensuite standardisé à $28 \mathrm{~g} / \mathrm{l}$ de matière grasse par de la crème ayant subi un traitement thermique de $115^{\circ} \mathrm{C} / 20 \mathrm{~s}$, puis stocké à $4^{\circ} \mathrm{C}$ pendant $36 \mathrm{~h}$ avant utilisation ;

- lot MFR (microfiltré réensemencé) : une fraction du lait MF (microfiltré standardisé), stockée $60 \mathrm{~h}$ à $4^{\circ} \mathrm{C}$, a èté inoculée par $0,01 \%(\mathrm{v} / \mathrm{v})$ d'une culture de $2 \mathrm{j}$ à $30^{\circ} \mathrm{C}$ de Propionibacterium freudenreichii subsp shermanii TL30.

Trois séries de fabrications de mini-fromages ont été réalisées, à $24 \mathrm{~h}$ d'intervalle, à partir d'un même lot de lait, ce qui nous a contraints à stocker ce lait à $4^{\circ} \mathrm{C}$ entre 12 et $60 \mathrm{~h}$ avant utilisation. Les mini-fabrications de fromage étaient conduites suivant le protocole habituel de l'Institut technique du gruyère (Buisson et al, 1987 ; ITG, 1991 ; 1993). Les levains commerciaux employés étaient composés d'une association de ferments thermophiles (Lactobacillus helveticus, Lactobacillus delbrueckii subsp lactis et Streptococcus salivarius subsp thermophilus) et de ferments mésophiles (Lactococcus lactis subsp lactis et Lactococcus lactis subsp cremoris).

Pour chaque lot de lait, trois mini-fromages ont été fabriqués à partir de $10 \mathrm{l}$ de lait chacun. Après saumurage, chaque fromage était découpé en 8 portions. Celles-ci étaient paraffinées et affinées dans des armoires thermostatées pendant $21 \mathrm{j}$ à $11^{\circ} \mathrm{C}$ (cave froide), puis $28 \mathrm{j}$ à $24^{\circ} \mathrm{C}$ (cave chaude). Au cours des fabrications, les contrôles habituels de coagulation, d'acidification et d'égouttage étaient pratiqués (ITG, 1991).

Pendant l'affinage en cave chaude, une portion de chaque fromage était prélevée au hasard. Elle était découpée en 2 échantillons, l'un pour l'analyse microbiologique immédiate, l'autre, congelé à $-18^{\circ} \mathrm{C}$, pour les analyses physicochimiques. Ces prélèvements étaient effectués après $1,3,5$, $7,10,14,21$ et $28 j$ de cave chaude.

\section{Analyses physicochimiques}

Le lactate a été dosé par méthode enzymatique (Boehringer Mannheim Meylan, France). Les teneurs en acides acétique et propionique ont été déterminées par chromatographie en phase gazeuse (Berdagué, 1986).

\section{Numérations bactériennes}

Des échantillons de $10 \mathrm{~g}$ de fromage ont été broyés au waring blender (Labo-Standa, Caen, France) dans $90 \mathrm{ml}$ de citrate de sodium $(2 \%$ $\mathrm{p} / \mathrm{v})$. La flore mésophile aérobie était dénombrée sur Plate Count Agar (PCA) (Biokar, Beauvais, France) après incubation $3 \mathrm{j}$ à $30^{\circ} \mathrm{C}$.

Les dénombrements de bactéries propioniques ont été effectués en parallèle sur les milieux YELA (Hettinga et al, 1968) et le milieu développé par Madec et al (1993), que nous avons appelé LGA (lithium-glycérol-agar) dans cette étude. Le $\mathrm{pH}$ des 2 milieux a èté ramené à 7,0 avant autoclavage $\left(121^{\circ} \mathrm{C}, 15 \mathrm{~min}\right)$. Les boîtes de Petri ont été incubées en anaérobiose (Anaerocult, Merck, Nogent sur Marne, France) à $30^{\circ} \mathrm{C}$ pendant $6 \mathrm{j}$.

Afin d'apprécier, pour les milieux YELA et LGA, d'une part, le caractère sélectif (croissance exclusive de la flore propionique) et d'autre part, la facilité de lecture des boîtes (différenciation des colonies formées par la flore propionique), 2 comptages distincts ont été effectués sur chaque milieu : la flore totale capable de se développer sur le milieu et la flore propionique. Les bactéries propioniques étaient dénombrées sur les 2 milieux par comptage des colonies de couleur crème de diamètre supérieur à $2 \mathrm{~mm}$. Sur LGA, l'observation d'un halo de décoloration traduisant la fermentation du glycérol (virage du pourpre de bromocrésol du violet au jaune) s'ajoute à ces 2 critères.

L'appartenance au genre Propionibacterium des souches utypiques" isolées sur YELA et LGA a été vérifiée par dosage HPLC de l'acide propionique produit (Boyaval et Corre, 1987) après culture sur YEL (YELA sans agar) pendant $48 \mathrm{~h}$. Les souches bactériennes productrices d'acide propionique ont été identifiées sur galeries API $50 \mathrm{CH}$ (Biomérieux, Marcy-l'Étoile, France). Les souches bactériennes non productrices d'acide propionique ont été identifiées grâce aux galeries API suivantes (Biomérieux, Marcy-l'Étoile, France) : API 20Strept (pour les coques par paires ou en chaînettes, Gram+, catalase-), API 20E (pour les bacilles Gram-) et API $50 \mathrm{CH}$ (pour les bacilles Gram+, catalase-, isolés ou en chainettes). 


\section{Électrophorèse en champs pulsés}

La différenciation des colonies de bactéries propioniques isolées à partir des fromages a été réalisée par comparaison des profils de restriction de I'ADN chromosomique obtenus après électrophorèse en champs pulsés dans les conditions opératoires suivantes : enzyme de restriction : Xbal, gel à $1 \%$ agarose, $6 \mathrm{~V} / \mathrm{cm}, 14^{\circ} \mathrm{C}$, rampe de 1 à $20 \mathrm{~s}$ pendant $20 \mathrm{~h}$ (Gautier et al,1992).

\section{RÉSULTATS}

\section{Analyses physicochimiques des fromages}

Les paramètres de fabrication sont en accord avec les valeurs habituelles relevées lors de ce type de fabrication après $30 \mathrm{~min}$ de pressage :

- extrait sec : de 57,1 à $59,6 \%$;

- extrait sec dégraissé : de 30,4 à $31,9 \%$;

- humidité/extrait sec dégraissé : de 1,26 à 1,41.

Le $\mathrm{pH}$ en fin d'acidification (à $20 \mathrm{~h}$ ) était compris entre 5,15 et 5,18 . L'évolution des teneurs en lactate, acétate et propionate des fromages issus de lait microfiltré correspondent aux valeurs habituellement enregistrées lors de ce type de fabrication (ITG, 1993).

Seuls les fromages fabriqués à partir de lait non microfiltré (TH) ou de lait réensemencé après microfiltration (MFR) présentent une ouverture, détectable respectivement après 1 et 2 semaines d'affinage en cave chaude.

\section{Croissance des bactéries propioniques}

Les figures $1 \mathrm{~A}, 1 \mathrm{~B}$ et $1 \mathrm{C}$ correspondant respectivement aux fabrications $\mathrm{TH}, \mathrm{MF}$ et MFR décrivent la cinétique de croissance pendant l'affinage en cave chaude des bactéries propioniques (dénombrées sur les milieux YELA et LGA) et de la flore totale dénombrable sur les milieux PCA, YELA et LGA. Les valeurs indiquées sont les valeurs moyennes des résultats obtenus pour les trois répétitions.

\section{Fabrication à partir de lait thermisé (lot TH, fig 1A)}

La flore aérobie mésophile et la flore propionique du lait de fabrication s'élevaient respectivement à $2,310^{4}$ et $2,410^{2} \mathrm{UFC} / \mathrm{ml}$. Le dénombrement des bactéries propioniques sur YELA et LGA a conduit à des résultats similaires. Les bactéries propioniques s'élevaient à $510^{2} \mathrm{UFC} / \mathrm{g}$ dans le caillé au moulage. Leur croissance n'a pas été discernable avant le début de l'affinage en cave chaude, puis leur nombre s'est accru rapidement à $10^{8} \mathrm{UFC} / \mathrm{g}$ après 2 semaines, pour atteindre $10^{9} \mathrm{UFC} / \mathrm{g}$ en fin d'affinage en cave chaude. La concentration en lactate décroît de 183 à $104 \mathrm{mmol} / \mathrm{kg}$ de fromage au cours de l'affinage en cave chaude. La production de propionate n'est pas détectable avant 2 semaines d'affinage et atteint $37 \mathrm{mmol} / \mathrm{kg}$ de fromage après 4 semaines en cave chaude.

\section{Fabrication à partir de lait thermisé microfiltré (lot MF, fig 1B)}

La flore totale du lait après microfiltration a été réduite de $2,310^{4} \mathrm{UFC} / \mathrm{ml}$ à environ 10 $\mathrm{UFC} / \mathrm{ml}$, ce qui correspond à un facteur de réduction décimale de 3,4 compris dans la fourchette de 2,12 à 3,63 obtenue par Trouvé et al (1991) pour divers micro-organismes du lait. Le stockage avant fabrication ( $36 \mathrm{~h}$ à $4^{\circ} \mathrm{C}$ ) du lait microfiltré a entraîné cependant une augmentation de sa flore totale à $510^{2} \mathrm{UFC} / \mathrm{ml}$.

La flore propionique est trop faible pour être dénombrée après microfiltration. L'application à la flore propionique initiale 
$\left(2,410^{2} \mathrm{UFC} / \mathrm{ml}\right)$ du facteur de réduction décimale de 2,18 obtenue pour Propionibacterium par Trouvé et al (1991) conduit à moins de 2 bactéries propioniques par
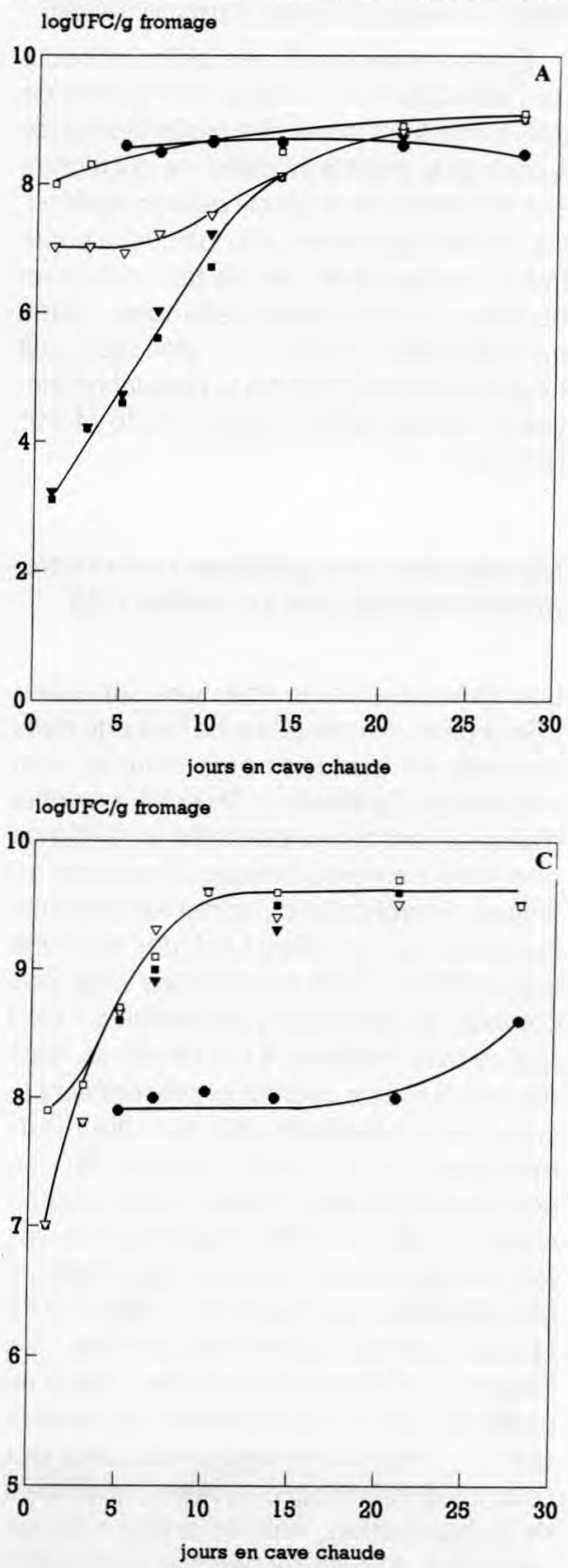

millilitre de lait microfiltré. Du fait du nombre très limité de bactéries propioniques en début d'affinage lors de cette fabrication, les premiers dénombrements ne sont pas fiables.

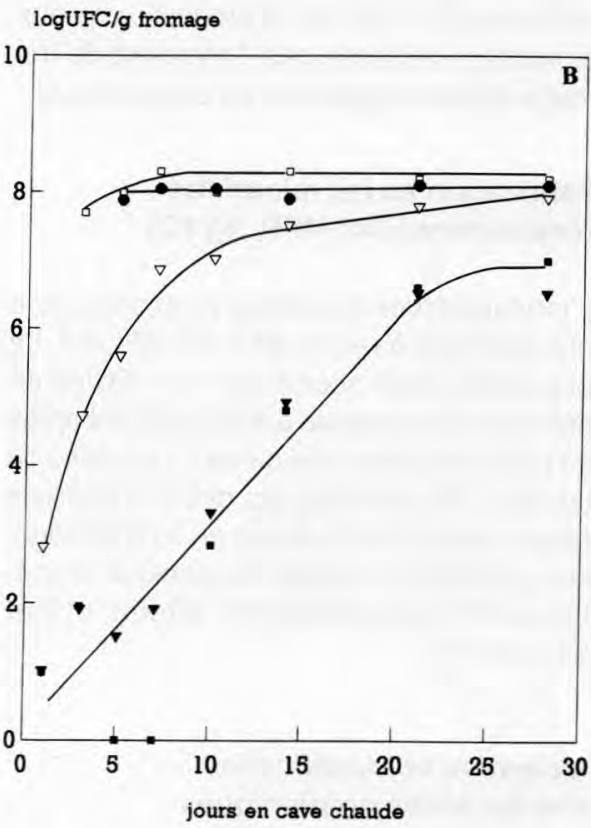

Fig 1. Croissance de différentes flores bactériennes pendant I'affinage en cave chaude de mini-fromages de type emmental. A : Fabriqué à partir de lait thermisé (TH) ; B : fabriqué à partir de lait thermisé microfiltré (MF) ; C : fabriqué à partir de lait thermisé microfiltré réensemencé par $P$ freudenreichii subsp shermanii (MFR). Flore totale dénombrable sur: $\bullet$ PCA ; $\square$ YELA ; $\nabla$ LGA ; flore propionique dénombrable sur : $\mathrm{YELA}$; $\nabla$ LGA.

Growth of different bacterial flora during ripening in warm room of experimental emmental cheeses made. A: From thermized milk (TH); $\boldsymbol{B}$ : from thermized microfiltered milk (MF); $\boldsymbol{C}$ : from thermized microfiltered milk inoculated with pure culture of $\mathrm{P}$ freudenreichii subsp shermanii (MFR). Total bacterial counts on: $P C A ; \square$ YELA; $\nabla L G A$; propionibacteria counts on: $\quad Y E L A ; \nabla L G A$. 
Après $10 \mathrm{j}$ en cave chaude, la flore propionique s'élevait à $10^{3} \mathrm{UFC} / g$ de fromage, sa croissance s'est effectuée de manière exponentielle jusqu'à environ $10^{6}$ puis a ralenti pour se stabiliser autour de $310^{6}$ $\mathrm{UFC} / \mathrm{g}$ de fromage. La teneur en lactate reste sensiblement égale à $150 \mathrm{mmol} / \mathrm{kg}$ de fromage, tandis que la production de propionate ne dépasse pas $3 \mathrm{mmol} / \mathrm{kg}$ de fromage après 4 semaines en cave chaude.

\section{Fabrication au lait microfiltré réensemencé (lot MFR, fig 1C)}

L'inoculation en bactéries propioniques a été effectuée à raison de $410^{5} \mathrm{UFC} / \mathrm{ml}$. La population s'est accrue de $10^{7} \mathrm{UFC} / \mathrm{ml}$ en début de cave chaude à $410^{9} \mathrm{UFC} / \mathrm{ml}$ après $10 \mathrm{j}$ et se stabilise à ce niveau. La totalité du lactate $(164 \mathrm{mmol} / \mathrm{kg}$ en début d'affinage cave chaude) a été utilisée en fin d'affinage, les productions d'acides propionique et acétique ont respectivement atteint 118 et $54 \mathrm{mmol} / \mathrm{kg}$.

\section{Isolement et identification des bactéries propioniques}

Cinquante à 100 colonies de bactéries propioniques ont été isolées au cours de l'affinage pour chaque fabrication, afin d'évaluer la diversité de la flore présente. Parmi les colonies isolées, les cultures présentant des caractères distincts lors de la croissance sur YEL ont été présélectionnées. L'analyse des profils de restriction d'ADN par électrophorèse en champs pulsés a été effectuée sur 16 colonies "sauvages" ainsi présélectionnées à partir d'une fabrication non ensemencée en bactéries propioniques (TH). Dix profils distincts ont pu être mis en évidence, ce qui laisse supposer qu'un grand nombre de souches sont présentes et se développent simultanément pendant l'affinage. Du fait du nombre réduit de colonies analysées par cette méthode, il n'a pas été possible de mettre en évidence la prédominance d'une ou plusieurs souches. Les profils biochimiques de ces dix souches, établis sur galeries API $50 \mathrm{CH}$, correspondent à ceux de l'espèce $P$ freudenreichii.

En revanche, les clones isolés au cours de l'affinage du fromage réensemencé (MFR) montrent toutes des profils identiques à celui de la souche inoculée, ce qui montre son développement effectif dans le fromage. Ce résultat était attendu du fait que la population résiduelle du lait de fabrication en bactéries propioniques "sauvages" après microfiltration ( $<10 \mathrm{UFC} / \mathrm{ml}$ ) était tout à fait négligeable par rapport à la population élevée du levain propionique inoculé $\left(410^{5}\right.$ UFC/ml).

\section{Identification des bactéries non propio- niques dénombrées sur milieu LGA}

Les dénombrements effectués sur milieu LGA à partir d'échantillons de fromage dans lesquels les bactéries propioniques sont minoritaires (fabrications TH et MF en début d'affinage) ont permis de mettre en évidence une flore non propionique résistante au lithium. Parmi celle-ci, certaines souches formaient sur le milieu LGA des colonies susceptibles d'être confondues avec des colonies de bactéries propioniques : bien que de taille inférieure à ces demières, elles avaient la même couleur et présentaient le caractère "acidifiant" sur ce milieu (fermentation du glycérol). Aucune de ces souches ne produit d'acide propionique en culture de $48 \mathrm{~h}$ sur YEL. Parmi les colonies les plus acidifiantes sur LGA, 6 souches ont été identifiées au moyen de galeries API. Quatre ont été identifiées comme des souches d' Enterococcus durans, une a un profil de Lactobacillus curvatus, la dernière étant un Enterobacter aerogenes. Deux des souches d'entérocoques isolées provenaient de la fabrication réalisée à partir de lait microfiltré. Afin de rechercher leur origine 
possible, des isolements ont été effectués sur milieu LGA à partir des levains lactiques utilisés en fabrication et de la saumure. Une souche d'Enterococcus durans a pu être isolée du levain de Lactobacillus helveticus ainsi que des souches identifiées comme Lactococcus lactis présentant également, mais à un moindre degré, le caractère "acidifiant" sur LGA. Une souche d'Enterococcus faecium a également été isolée de la saumure.

\section{DISCUSSION}

\section{Comparaison des milieux YELA et LGA}

\section{Sélectivité}

Lorsque les bactéries propioniques sont majoritaires, la flore totale dénombrée sur YELA et LGA correspond à la flore propionique et se situe à un niveau supérieur à la flore totale dénombrée sur PCA, sur lequel les bactéries propioniques ne se développent pas dans les conditions d'incubation utilisées (aérobiose).

En revanche, lorsque la population de bactéries propioniques n'est pas dominante, le dénombrement de la flore totale poussant sur YELA $\left(6 \mathrm{j}\right.$ à $30^{\circ} \mathrm{C}$ en anaérobiose $)$ a conduit aux mêmes valeurs que le dénombrement sur PCA $\left(3 \mathrm{j}\right.$ à $30^{\circ} \mathrm{C}$ en aérobiose), ce qui met en évidence l'absence de sélectivité du milieu YELA vis-à-vis des micro-organismes du fromage. Compte-tenu des valeurs élevées trouvées dans les fabrications effectuées à partir de lait microfiltré, il est vraisemblable que cette flore est principalement composée de bactéries lactiques aéro-anaérobies facultatives provenant des levains, capables de se développer dans différentes conditions d'incubation (Baird et al, 1987).

Pour les 3 fabrications, la flore totale dénombrable sur LGA a été inférieure de 1 à 2 logarithmes décimaux à la flore totale poussant sur YELA, ce qui traduit un degré de sélectivité supérieur du milieu LGA.

Cet écart de dénombrement de flore totale entre les 2 milieux peut s'élever (jusqu'à 3,5 logarithmes décimaux), en particulier en début d'affinage pour les fromages fabriqués à partir de lait microfiltré non ensemencé en bactéries propioniques (essai MF) où la flore dominante n'est constituée en début d'affinage que par les levains lactiques. Ceci traduit le fait que les bactéries lactiques mises en œuvre en fabrication d'emmental ne se développent pas sur le milieu LGA et confirme les résultats obtenus par Madec et al, (1993) avec des cultures pures de diverses bactéries lactiques.

\section{Différenciation des colonies de bactéries propioniques}

Pour les 3 fabrications, le dénombrement des bactéries propioniques sur YELA et sur LGA a conduit à des résultats similaires. II semble cependant que le milieu LGA présente un meilleur seuil de détection, en améliorant la "lecture" des boîtes lorsque la flore propionique présente dans l'échantillon est très basse. C'est le cas des dénombrements effectués en tout début d'affinage sur les fromages MF, pour lesquels la population propionique est inférieure à $100 \mathrm{UFC/g}$ de fromage. Dans le lait de fabrication et le caillé frais, le dénombrement des bactéries propioniques se révèle très délicat sur les 2 milieux. En effet, dans la pratique, le critère de décoloration décrit pour la reconnaissance des bactéries propioniques sur LGA (Madec et al, 1993), à savoir l'observation de halos de décoloration autour des colonies de bactéries propioniques n'est pas toujours utilisable : selon le niveau et la nature de la flore résistante au lithium poussant sur ce milieu, la totalité de la culture peut virer au violet pâle; la difficulté de "lecture" des milieux LGA et YELA est alors 
identique. Par ailleurs, des colonies de bactéries non propioniques présentant le caractère "acidifiant", bien qu'étant dénombrées à des dilutions 100 à 10000 fois plus fortes que les bactéries propioniques, peuvent entraîner des dénombrements erronés si des précautions ne sont pas prises. Ces "faux-positifs" sont observés tant que la population propionique n'est pas dominante (fabrication MF et 10 premiers jours d'affinage en cave chaude pour la fabrication TH). L'aptitude à fermenter le glycérol, peu répandue parmi les bactéries lactiques, est cependant retrouvée chez certains lactobacilles mésophiles (Zanoni et al, 1987 ; Collins et al, 1989) et entérocoques (Mundt, 1986). Ces micro-organismes font partie de la flore capable de se développer pendant l'affinage des pâtes pressées cuites (Sharpe, 1962 ; Efthymiou et al, 1974). L'origine de cette flore secondaire, parmi laquelle les entérocoques semblent tenir une place prépondérante, n'a pas été déterminée de façon certaine. La présence d'entérocoques dans un des levains commerciaux employé et dans la saumure peut expliquer cependant le fait que des souches d'Enterococcus durans aient été retrouvées dans les fromages fabriqués à partir de lait microfiltré, dans lequel la flore totale n'excédait pas $510^{2} \mathrm{UFC} / \mathrm{ml}$. La résistance thermique des entérocoques (Sanz Perez et al, 1982) peut expliquer leur présence dans les fromages fabriqués à partir de lait thermisé.

En conclusion, le milieu LGA a une sélectivité élevée vis-à-vis des levains lactiques mais présente les limites d'utilisation qui viennent d'être décrites pour dénombrer les bactéries propioniques dans les fromages fabriqués à partir de lait peu épuré (thermisé), dans lesquels une flore complexe, provenant du lait, se développe pendant l'affinage. Cette constatation a donné lieu à l'amélioration de la sélectivité du milieu LGA vis-à-vis de la flore du lait (Madec et al, 1994).

\section{Croissance des bactéries propioniques}

Les principales données sur la cinétique de croissance des bactéries propioniques pendant l'affinage de fromages expérimentaux de type emmental ont été comparées (tableau I). Les populations finales atteintes par les bactéries propioniques sont respectivement de 9,0 et $9,5 \mathrm{log}$ UFC/g pour les fabrications effectuées au lait thermisé (TH) et microfiltré réensemencé (MFR). Ces niveaux de population sont comparables à ceux qui ont été reportés dans le cas de fromages expérimentaux (Hettinga et al, 1974 ; Fryer et Peberdy, 1977 ; Gilles et al, 1983 ; Turner et al, 1983 ; Baer et al, 1993) ou de fromages commerciaux (Steffen et al, 1986). Ces niveaux de population ne sont cependant pas atteints au même stade d'affinage en cave chaude pour les fabrications TH et MFR, mais après respectivement 2 et 4 semaines.

L'analyse des profils de restriction d'ADN confirme que seul le levain propionique mis en œuvre se développe dans les fromages MFR. Les taux de croissance des bactéries propioniques ont été très voisins (respectivement 18,6 et 18,1 ) pour les 2 fabrications TH et MFR, bien qu'il s'agisse de 2 populations très différentes dans chaque cas : une culture "sauvage" multi-souches dans le cas de la fabrication $\mathrm{TH}$, une culture pure de $P$ freudenreichii subsp shermanii TL 30 dans le cas de la fabrication MFR.

Dans le cas des fromages fabriqués à partir de lait microfiltré non réensemencé en bactéries propioniques (MF), la croissance des bactéries propioniques est plus lente (temps de génération égal à 25,6 h), la population finale atteinte par les bactéries propioniques n'est que de $6,8 \mathrm{log} U F C / g$ et la production d'acide propionique se situe à la limite de détection. L'essai MF n'a malheureusement pas pu être prolongé pour confirmer la stabilisation de la flore propionique à ce niveau. 
Tableau I. Cinétique de croissance des bactéries propioniques au cours de l'affinage en cave chaude de fabrications expérimentales de type emmental.

Growth of propionibacteria during warm room ripening of experimental Swiss-type cheeses.

\begin{tabular}{|c|c|c|c|c|c|c|c|c|}
\hline \multicolumn{3}{|c|}{ Paramètres de fabrication } & \multicolumn{4}{|c|}{ Croissance Propionibacterium } & \multirow{3}{*}{$\operatorname{Tg}(h)$} & \multirow[t]{3}{*}{ Référence } \\
\hline \multirow{2}{*}{$\begin{array}{l}\text { Traitement } \\
\text { lait }\end{array}$} & \multirow{2}{*}{$p H$ à $24 h$} & \multirow{2}{*}{$\begin{array}{c}\text { Température } \\
\text { cave chaude } \\
{ }^{\circ} \mathrm{C}\end{array}$} & \multirow{2}{*}{$\begin{array}{l}\text { Inoculum } \\
(\mu / / \text { lait })\end{array}$} & \multicolumn{3}{|c|}{$\log U F C / m l$} & & \\
\hline & & & & entrée CC & milieu CC & fin $C C$ & & \\
\hline $\mathrm{TH}$ & 5,17 & 24 & 0 & 2,8 & 8,1 & 9,0 & 18,8 & Cette étude \\
\hline MF & 5,17 & 24 & 0 & $<1$ & 4,6 & 6,8 & 25,6 & Cette étude \\
\hline MFR & 5,15 & 24 & 100 & 6,5 & 9,5 & 9,5 & 18,1 & Cette étude \\
\hline$P$ & (1)- & - & - & 6,4 & 9,4 & & 18\# & Hettinga et al, 1974 \\
\hline$P$ & 5,37 & $21-22$ & 75 & 6,2 & 8,1 & 9,0 & 41\# & Fryer \& Peberdy, 1977 \\
\hline$P$ & $5,15-5,30$ & 22 & 15 & 6,5 & 8,7 & 9,0 & $46 \#$ & Gilles et al, 1983 \\
\hline$P$ & 5,0 & 22 & 15 & 6,3 & 9,0 & - & 25\# & Turner et al, 1983 \\
\hline- & - & - & $0,0005 \#$ & $<1$ & 6,8 & 9,1 & 30\# & Baer et al, 1993 \\
\hline & & & $0,083 \#$ & 3,2 & 8,8 & 9,2 & 35\# & Baer et al, 1993 \\
\hline & & & $5 \#$ & 5,4 & 8,8 & 9,4 & 38\# & Baer et al, 1993 \\
\hline
\end{tabular}

TH, MF, MFR : voir texte ; P: pasteurisé ; CC : cave chaude ; entrée : 0 ou $1 \mathrm{j}$; milieu : $14 \mathrm{j}$; fin : $28 \mathrm{j}$; sauf pour Baer et al, $1993: 5,45 \mathrm{et} 90 \mathrm{j}$. Tg : temps de génération ; \# calculé d'après les données des auteurs (hypothèse : inoculum à $9,7 \mathrm{ufc} / \mathrm{ml}$ ) ; (1) - : donnée non disponible.

TH, MF, MFR: see text; P: pasteurized; CC: warm room; entrée: 0 to 1 d; milieu: 14 d; fin: 28 d; except for Baer et al, 1993: 5, 45 and 90 d. Tg: generation time; \# calculated from authors data (hyp: inoculum at $9.7 \log$ cfu/ml); (1) -: not available data. 
La différence entre les temps de génération observés pour cette fabrication d'une part et les essais TH et MFR d'autre part, peut être due au développement d'une flore contaminante inhabituelle susceptible d'inhiber le développement des bactéries propioniques ou à la nature de la population propionique présente ou encore à une combinaison de ces facteurs.

Les valeurs des temps de génération obtenus pendant l'affinage des fromages TH et MFR sont voisines de celles que nous avons calculées à partir des données obtenues par Hettinga et al (1974) et Turner et al (1983) lors de fabrications de fromages emmental ensemencés avec une souche de $P$ freudenreichii subsp shermanii. En revanche, dans d'autres fabrications expérimentales d'emmental décrites par Fryer et Peberdy (1977), Gilles et al (1983) et Baer et al (1993), ensemencées à différents niveaux par $P$ freudenreichii, les temps de génération des bactéries propioniques observés en cave chaude $\left(21-22^{\circ} \mathrm{C}\right)$, compris entre 30 et $46 \mathrm{~h}$, étaient supérieurs à ceux obtenus dans cette étude.

Les connaissances concernant les cinétiques de croissance des bactéries propioniques dans le fromage sont limitées. Cependant, les différences considérables de comportement des bactéries propioniques en milieu liquide et la connaissance de la sensibilité de certaines souches à des facteurs tels que de faibles différences de $\mathrm{pH}$ autour de 5,0-5,5 (Langsrud et Reinbold, 1973) peuvent suffire pour rendre compte des variations observées du taux de croissance.

\section{RÉFÉRENCES}

Baer A, Ryba I, Grand M (1993) Ursachen der Entstehung von braunen Tupfen im Käse. Schweiz Milchw Forschung 22, 3-7

Baird RM, Corry JEL, Curtis GDW (1987) Pharmacopoeia of culture media for food microbiology. Int J Food Microbiol 5, 187-300
Berdagué JL (1986) Méthode d'extraction rapide des acides gras volatils des fromages. Lait $66,223-246$

Boyaval P, Corre C (1987) Continuous fermentation of sweet whey permeate for propionic acid production in a CSTR with UF recycle. Biotechnol Lett 9, 801-806

Britz TJ, Holzapfel WH (1973) The suitability of different media for the isolation and enumeration of propionibacteria from dairy products. S Afr J Dairy Technol 5, 213-216

Buisson V, Kerjean JR, Courrove M (1987) Miniaturisation de la fabrication fromagère, un nouveau test pour mesurer l'aptitude fromagère des laits. Tech Lait 1024, 17-23

Collins MD, Phillips BA, Zanoni P (1989) Deoxyribonucleic acid homology studies of Lactobacillus casei, Lactobacillus paracasei sp nov, subsp paracasei and subsp tolerans, and Lactobacillus rhamnosus sp nov, comb nov. Int J Syst Bacteriol 39, 105-108

Drinan FD, Cogan TM (1992) Detection of Propionibacteria in cheese. J Dairy Res 59, 1-5

Efthymiou CJ, Baccash P. Labombardi VJ, Epstein DS (1974) Improved isolation and differentiation of enterococci in cheese. Appl Microbiol 28, 417-422

Fryer TF, Peberdy MF (1977) Growth of propionibacteria in Swiss and Egmont cheese. $N Z$ J Dairy Sci Technol 12, 133-134

Gautier M, Mouchel N, Rouault A, Sanseau P (1992) Determination of genome size of four Propionibacterium species by pulsed-field gel electrophoresis. Lait 72, 421-426

Gilles J, Turner KW, Martley FG (1983) Swisstype cheese. I. Manufacturing and sampling procedures. NZ J Dairy Sci Technol 18, 109-115

Hettinga DH, Vedamuthu ER, Reinbold GW (1968) Pouch method for isolating and enumerating propionibacteria. J Dairy Sci 52, 1707-1709

Hettinga DH, Reinbold GW, Vedamuthu ER (1974) Split defect of Swiss cheese I. Effect of strain of propionibacteria and wrapping material. J Milk Food Technol 37, 322-328

ITG (1991) Minifabrication de fromages à pâte pressée cuite : les choix de l'ITG depuis 1987. "Etude ITG/ZO/1991/02/B (Edition ITG, LaRoche-sur-Foron, France)

ITG (1993) Aptitude technologique de bactéries propioniques laitières. Étude en minifabrica- 
tion. Étude ITG/ZO/1993, sous presse (Edition ITG, La Roche sur Foron, France)

Kurmann J (1962) Eine Metodische Verbesserung zur Bestimmung des Propionsäurebakteriengehaltes der Emmentalerkäsereimilch. XVI Int Dairy Congr C, 377-382

Langsrud T, Reinbold GW (1973) Flavor development and microbiology of Swiss cheese. A review. I. Milk qualities and treatments. J Milk Food Technol 36, 487-490

Madec MN, Rouault A, Maubois JL (1993) Milieu sélectif et procédé pour le dénombrement des bactéries propioniques. Brevet $\mathrm{Fr} n^{\circ}$ dépot 9300823

Madec MN, Rouault A, Maubois JL, Thierry A (1994) Milieu sélectif pour le dénombrement des bactéries propioniques. Brevet européen $n^{\circ}$ dépot PCT/FR94 00082

Mundt JO (1986) Enterococci. In: Bergey's manual of systematic bacteriology (Sneath $\mathrm{PH}$, Mair NS, Sharpe HE, Holt JG, eds). The Williams and Wilkins Company, Baltimore, vol 2, 1063-1065

Peberdy MF, Fryer TF (1976) Improved selective media for the enumeration of propionibacteria from cheese. NZ J Dairy Sci Technol 11, 10-15
Sanz Perez B, Lopez Lorenzo P, Garcia ML, Hernandez PE, Ordonez JA (1982) Heat resistance of enterococci. Milchwissenschaft 37 , 724-726

Sharpe ME (1962) Enumeration and studies of Lactobacilli in food products. Dairy Sci Abstr 24, 165-171

Steffen C, Flueckiger E, Bosset JO, Ruegg M (1986) Swiss-type varieties. In: Cheese : chemistry, physics and microbiology. Major cheese groups, vol 2 (Fox PF ed). Elsevier, London, 93-120

Tomes NJ, Henderick CA, Glatz BA (1991) Selective medium for Propionibacterium growth. US Pat $\mathrm{n}^{\circ} 5026647$

Trouvé E, Maubois JL, Piot M, Madec MN, Fauquant J, Rouault A, Tabard J, Brinkman G (1991) Rétention de différentes espèces microbiennes lors de l'épuration du lait par microfiltration en flux tangentiel. Lait 71, 1-13

Turner KW, Morris HA, Martley FG (1983) Swisstype cheese. II.The role of thermophilic lactobacilli in sugar fermentation. NZ J Dairy Sci Technol 18, 117-123

Zanoni $P$, Farrow JAE, Phillips BA, Collins MD (1987) Lactobacillus pentosus (Fred, Peterson and Anderson) sp nov, nom rev. Int $J$ Syst Bacteriol 37, 339-341 